\title{
Distinctions in Weight Perception between Constrained and Unconstrained Lifting of Objects
}

\author{
S. M. Mizanoor Rahman ${ }^{1,}$, Ryojun Ikeura, b , Soichiro Hayakawa ${ }^{2}$, \\ Haoyong $\mathrm{Yu}^{1}$ \\ ${ }^{1}$ Division of Bioengineering, Faculty of Engineering, National University of Singapore \\ ${ }^{2}$ Division of Mechanical Engineering, Graduate School of Engineering, Mie University, Tsu, Japan \\ amizansm@hotmail.com; bikeura@ss.mach.mie-u.ac.jp
}

Keywords: Human, Weight Perception, Object Lifting, Constrained Lifting, Unconstrained Lifting, Psychophysics, Assistive Robotics, Safe Human-Robot Interaction

\begin{abstract}
We made two objects (hollow metal cylinders) with identical appearance. One was called the constrained object; another was called the unconstrained object. Human subjects lifted the constrained and unconstrained objects separately for various experiment conditions. We compared human's weight perception between the two types of objects. Results showed that weight perception between constrained and unconstrained lifting was almost the same. In our previous research, we developed a power assist system for lifting objects and determined a psychophysical relationship between actual and perceived weights for the objects lifted with the system [1]. The perceived weights were $40 \%$ of actual weights. However, the objects lifted with the system were constrained objects (as they were tied to the force sensor) that might affect the accuracy of the relationship. The results of this paper confirm that the constraint does not affect weight perception too much, which reconfirms that the psychophysical relationship between actual and perceived weights derived in our previous research was correct [1].
\end{abstract}

\section{Introduction}

Power assist robots are mainly used for rehabilitation and health care purposes [2], [3]. We tried to bring novelty in the application of the power assist robots in [1]. In [1], we argued that power assist systems could be used to handle heavy objects in various industries. There are few models of power assist robots for objects manipulation [4], [5]. However, these models do not include human features such as human's weight perception in their controls and thus these models are not so human-friendly. We proposed a weight-perception-based model of power assist robot system for lifting heavy objects in various industries in [1].

In [1], we determined a psychophysical relationship between the actual weights and the perceived weights for lifting objects with a power assist robot system. Subjects lifted the power-assisted objects with the power assist robot and then compared the perceived weights to the reference weights and then determined the psychophysical relationship. However, the power-assisted object was a constrained object because it was tied to a force sensor. On the other hand, the actual object (reference weight object) was lifted manually and was not tied to any force sensor, and hence it was an unconstrained object. We assumed that the difference in constrained and unconstrained lifting conditions might affect the accuracy of the psychophysical relationship. This paper aims to investigate whether there is any difference in human's weight perception between constrained and unconstrained lifting of the same objects.

In this paper, we made two objects (hollow metal cylinders) with identical appearance. One was called the constrained object; another was called the unconstrained object. Human subjects lifted the constrained and unconstrained objects separately for various experiment conditions. We compared human's weight perception between the two types of objects. Results showed that weight perception between constrained and unconstrained lifting was almost the same, which reconfirms that the psychophysical relationship between actual and perceived weights derived in [1] was correct. 


\section{Materials}

We made two objects (hollow metal cylinders, hollow cylinder 1 and hollow cylinder 2) with identical appearance. The cylinders are shown in Fig.1(a) and details are presented in Table 1. A solid metal stick (height: $13 \mathrm{~cm}$, diameter: $2 \mathrm{~cm}$ ) was also made and vertically fixed on a table as

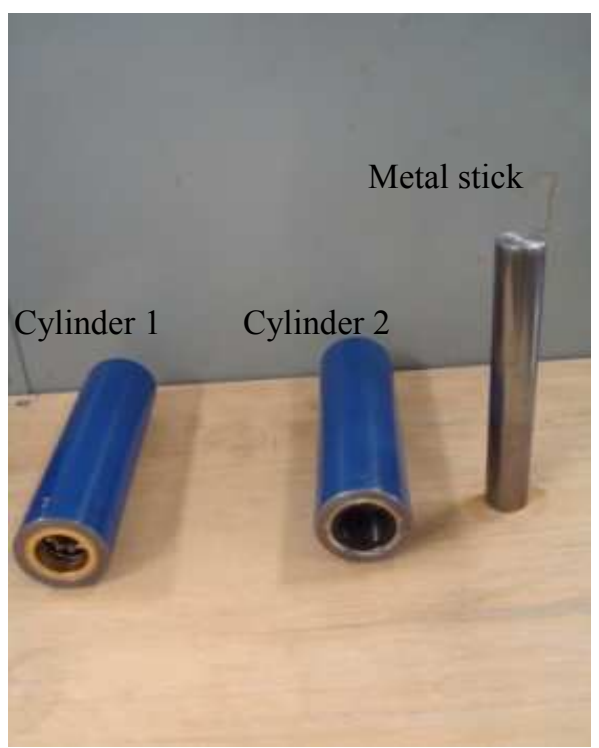

(a)

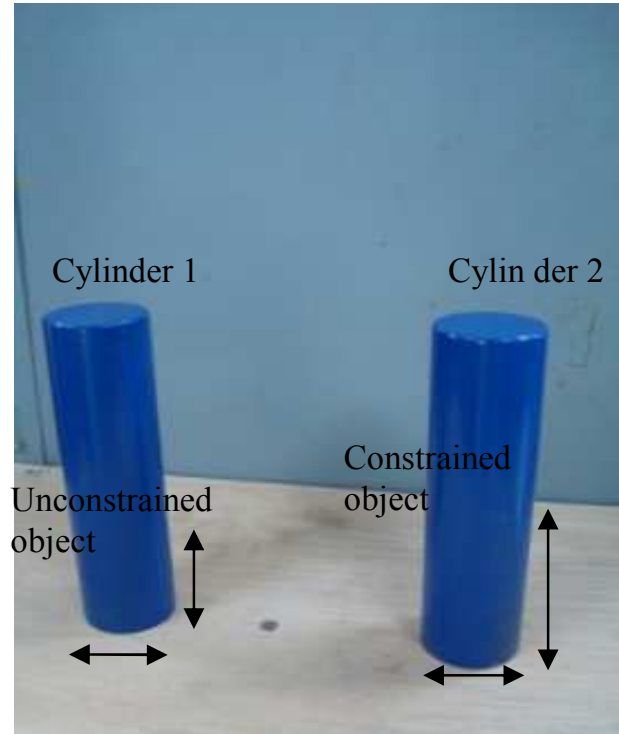

(b)

Fig. 1 Configuration of constrained (cylinder 2 with metal stick inside) and unconstrained (cylinder 1) objects.

shown in Fig.1 (a). The hollow cylinder 2 was vertically placed on the table and the metal stick was inside the cylinder as shown in Fig.1(b). This arrangement restricted the movement of the cylinder 2 along the horizontal direction and thus made it a contrained object. The constrained object could be moved (lifted) only along the vertical direction. The hollow cylinder 1 was vertically placed on the table as shown in Fig.1(b) and there was nothing inside it. Hence, it was an unconstrained object and it could be moved along horizontal and vertical directions. The constrained and the unconstrained objects were put on the same table and were very close to each other.

Table 1 : Details of Cylinder 1 and Cylinder 2

\begin{tabular}{ccc}
\hline Items & Cylinder 2 & Cylinder 1 \\
\hline Weight & $910 \mathrm{~g}$ & $910 \mathrm{~g}$ \\
Height & $15 \mathrm{~cm}$ & $15 \mathrm{~cm}$ \\
Diameter (outer) & $4 \mathrm{~cm}$ & $4 \mathrm{~cm}$ \\
Diameter (inner) & $2.2 \mathrm{~cm}$ & $2.2 \mathrm{~cm}$ \\
\hline
\end{tabular}

\section{Methods}

Eight mechanical engineering male students aged between 21 and 31 years performed as the subjects. The subjects were physically and mentally healthy. They did not have any prior experience of this experiment method. Experiment procedures were instructed to the subjects, but no formal training was arranged for them.

In each trial, the subject lifted the constrained object as shown in Fig.2 (a). The task required the subject to lift the object approximately $0.1 \mathrm{~m}$, maintain the lift for $1-2$ seconds and then release the object. The subject then lifted the unconstrained object following the same procedure as employed for the constrained object. See Fig.2 (b). The experiment was conducted by all subjects separately. In each trial, each subject grasped (and lifted) the object at three distinct positions separately as follows:

(i) at the center of the object,

(ii) at the upper end,

(iii) at the lower end. 
Again, for each position, the subject used:

(i) power grip,

(ii) 3-finger grip.

Thus the subject compared the perceived weight of the constrained object to that of the unconstrained object for each trial. The weight of the unconstrained object was considered as the reference weight and its weight was assumed as the $100 \%$. The subject estimated the perceived weight of the constrained object comparing it to that of the reference weight. The subject was asked:

If the reference weight (unconstrained object weight) is assumed as $100 \%$, then what is the $\%$ for the perceived weight of the constrained object compared to the reference weight?

Subject's response regarding the perceived weight of the constrained object was recorded for each trial separately.

\section{Results}

Responses of each subject regarding the perceived weight of the constrained object in comparison with that of the unconstrained object for different experiment protocols are shown in Table 2 .

We see that the variation in the perceived weight of the constrained object with respect to the reference weight is not more than $10 \%$. However, a subject needs more than a $10 \%$ difference in perceived weights to be able to discriminate between the weights of two objects [6]. The results show that human's weight perception for constrained lifting does not vary too much in comparison

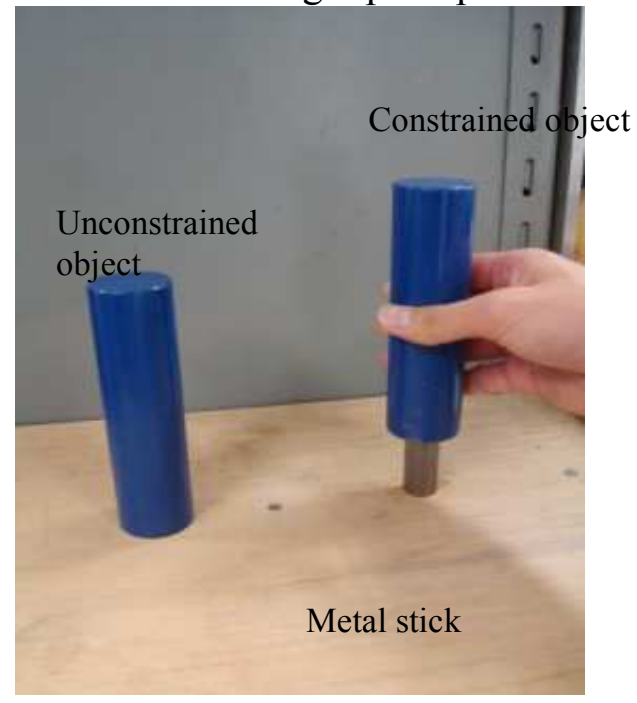

(a)

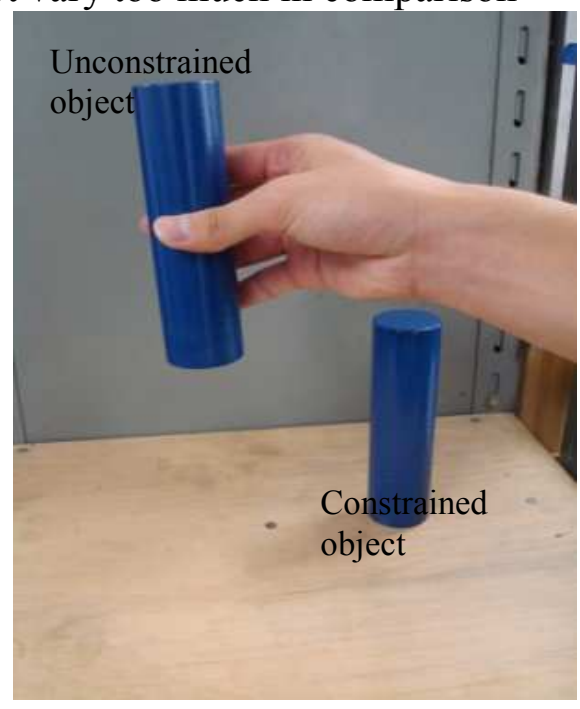

(b)

Fig.2 Lifting constrained and unconstrained objects by a human subject.

with that for the unconstrained lifting of the same object. The reasons may be that: (i) the total efforts for lifting the constrained object do not vary too much in comparison with that for the unconstrained object [7], (ii) object's visual cues were the same for both objects that resulted in similar weight perception, (iii) the inner metal stick did not touch the inner surface of the constrained object, hence the grip and the load force requirements did not vary between the constrained and the unconstrained lifting that resulted in similar weight perception, (iv) human's haptic cues, grasping method, posture, hand stiffness etc. did not vary between the constrained and the unconstrained lifting etc.[7].

Again, perceived weight for grasping at upper end or at lower end is more than that for grasping at the center of the object. The reason may be that gravity is sensed more intensely when grasped either at lower or at upper end than grasped at the centre.Similarly, the 3-finger grasp produced higher perceived weight than the power grip. The similar reason applies for this that the gravity is sensed more intensely when grasped with 3-fingers than when grasped with power grip. 
Table 2: Estimated weight of the constrained object in comparison with the reference weight for different experiment protocols.

\begin{tabular}{|c|c|c|c|c|c|c|c|}
\hline \multirow[t]{3}{*}{ Subjects } & \multirow{3}{*}{$\begin{array}{l}\text { Weight of } \\
\text { unconstrained } \\
\text { object } \\
\text { (reference } \\
\text { weight) }\end{array}$} & \multicolumn{6}{|c|}{ Estimated weight of constrained object } \\
\hline & & \multicolumn{2}{|c|}{ Grasped at centre } & \multicolumn{2}{|c|}{ Grasped at upper end } & \multicolumn{2}{|c|}{ Grasped at lower end } \\
\hline & & Power grip & 3-finger grip & Power grip & 3-finger grip & Power grip & 3-finger grip \\
\hline 1 & $100 \%$ & $95 \%$ & $100 \%$ & $105 \%$ & $110 \%$ & $105 \%$ & $110 \%$ \\
\hline 2 & $100 \%$ & $100 \%$ & $105 \%$ & $105 \%$ & $110 \%$ & $105 \%$ & $110 \%$ \\
\hline 3 & $100 \%$ & $95 \%$ & $100 \%$ & $105 \%$ & $110 \%$ & $105 \%$ & $110 \%$ \\
\hline 4 & $100 \%$ & $100 \%$ & $100 \%$ & $105 \%$ & $105 \%$ & $105 \%$ & $105 \%$ \\
\hline 5 & $100 \%$ & $100 \%$ & $105 \%$ & $110 \%$ & $110 \%$ & $110 \%$ & $110 \%$ \\
\hline 6 & $100 \%$ & $105 \%$ & $110 \%$ & $110 \%$ & $110 \%$ & $110 \%$ & $110 \%$ \\
\hline 7 & $100 \%$ & $95 \%$ & $100 \%$ & $105 \%$ & $110 \%$ & $105 \%$ & $110 \%$ \\
\hline 8 & $100 \%$ & $95 \%$ & $105 \%$ & $105 \%$ & $105 \%$ & $105 \%$ & $105 \%$ \\
\hline
\end{tabular}

\section{Conclusions and Future Works}

As presented in this paper, the human subjects lifted the constrained and the unconstrained objects separately for various experiment conditions and protocols. We compared human's weight perception between the two types of objects. Results showed that weight perception between the constrained and the unconstrained lifting did not vary too much, which reconfirms that the psychophysical relationship between actual and perceived weights for lifting objects with power-assist derived in our previous research in [1] was correct. In near future, we will develop appropriate control methods and strategies for power assist robots for manipulating heavy objects in industries based on the findings of this paper and of [1], [8]-[10].

\section{References}

[1] S.M.M.Rahman, R. Ikeura, M. Nobe, S. Hayakawa, H. Sawai, "Weight-perception-based model of power assist system for lifting objects," International Journal of Automation Technology, Vol.3, No.6, pp. 681-691, Nov 2009.

[2] S. Tashiro and T. Murakami, "Step passage control of a power-assisted wheelchair for a caregiver," IEEE Transactions on Industrial Electronics, Vol. 55, No. 4, April 2008, pp. $1715-1721$.

[3] H. Seki, K. Ishihara and S. Tadakuma, "Novel regenerative braking control of electric power-assisted wheelchair for safety downhill road driving," IEEE Trans. on Industrial Electronics, Vol. 56, No. 5, May 2009, pp. 1393-1400.

[4] T.Takubo,H. Arai,K. Tanie,T.Arai,"Human-robot cooperative handling using variable virtual nonholonomic constraint," International Journal of Automation Technology, Vol.3, No.6, pp. 653-662, 2009.

[5] A. Niinuma, T.Miyoshi, K. Terashima, Y. Miyashita, "Evaluation of effectiveness of a power-assisted wire suspension system compared to conventional machine," in Proceedings of 2009 IEEE International Conference on Mechatronics and Automation, August 9-12, 2009, pp.369-374.

[6] Y. Koike, J. Kim, S. Duk, "Role of stiffness in weight perception," Japanese Psychological Research, 2006, 48 (3), 174-187.

[7] J.R. Flanagan and C.A. Bandomir, "Coming to grips with weight perception: effects of grasp configuration on perceived heaviness," Perception \& Psychophysics, Vol. 62, No. 6, pp. 1204-1219, 2000.

[8] S.M.M.Rahman, R. Ikeura, S. Hayakawa, H. Sawai, "Design and control of a power assist system for lifting objects based on human operator's weight perception and load force characteristics," IEEE Transactions on Industrial Electronics, Vol.58, No.8,pp.3141-3150, August 2011.

[9] S.M.M.Rahman, R. Ikeura, S. Hayakawa, and H. Sawai, "Design guidelines for power assist robots for lifting heavy objects considering weight perception, grasp differences and worst-cases," International Journal of Mechatronics and Automation, Vol. 1, No. 1, pp.46-59, March 2011.

[10] S.M.M.Rahman, R. Ikeura, H. Sawai, "Design and control of a power-assist robot system for lifting objects based on human's unimanual and bimanual weight discrimination," International Journal of Assistive Robotics and Systems, Vol.11, No.2, pp.11-29, June 2010. 